\title{
O Manifesto dos filósofos alemães contra a psicologia experimental: introdução, tradução e comentários
}

\section{The Manifesto of German philosophers against experimental psychology: introduction, translation and commentaries}

\author{
Saulo de Freitas Araujo* \\ Universidade Federal de Juiz de Fora - UFJF, Juiz de Fora, Minas Gerais, Brasil
}

\begin{abstract}
RESUMO
Embora a psicologia tenha já há muito se separado institucionalmente da filosofia por todo o mundo, a história dessa separação é ainda pouco conhecida tanto por psicólogos quanto por filósofos. No entanto, diversamente do que se possa imaginar, essa separação não ocorreu de forma homogênea em todos os países. Ao contrário, ela reflete as particularidades de cada contexto cultural em questão. O objetivo deste artigo é contextualizar, em linhas gerais, um dos momentos decisivos na relação entre a filosofia e a psicologia na tradição alemã, que contribuiu para a posterior separação institucional de ambas naquela cultura: o Manifesto dos filósofos alemães, em 1913, contra a ocupação de cátedras de filosofia por psicólogos experimentais. Ao lado da tradução integral do manifesto, pela primeira vez publicada em português, será oferecida uma introdução e comentários gerais sobre o contexto envolvendo a preparação e as consequências do referido documento.
\end{abstract}

Palavras-chave: Filosofia alemã, Psicologia experimental, História da psicologia.

\begin{abstract}
Although psychology has long since been institutionally separated from philosophy throughout the world, the history of this separation is still poorly understood by both psychologists and philosophers. However, contrary to what one can imagine, this separation did not occur in a homogeneous way in all countries. Rather, it reflects the peculiar characteristics of each cultural context in question. The aim of this paper is to contextualize, in general lines, one of the defining moments in the relationship between philosophy and psychology in the German tradition, which later contributed to their institutional separation within that culture: the petition of German philosophers, in 1913, against the occupation of philosophical chairs by experimental psychologists. Beside the full translation of the manifesto, for the first time published in Portuguese, an introduction as well as general comments about the context surrounding the preparation and the consequences of that document will be offered.
\end{abstract}

Keywords: German philosophy, Experimental psychology, History of psychology. 


\section{I ntrodução}

Que a psicologia contemporânea esteja radicalmente separada da filosofia é um fato bem sabido por todos e até comemorado por alguns; que a psicologia tenha sido durante muito tempo parte integrante dos sistemas e institutos filosóficos também não é novidade para nenhum filósofo ou psicólogo. No entanto, o que é muito pouco conhecido por ambos os lados é exatamente o contexto dessa transformação. Como e por que se deu essa separação?

Antes de tudo, é preciso reconhecer que a relação entre a filosofia e a psicologia, sobretudo no que diz respeito aos seus aspectos institucionais, apresenta contornos específicos em cada um dos contextos sócio-culturais em que ela aparece. Por exemplo, na Alemanha, nos EUA e na França, o desenvolvimento e a consolidação da psicologia como ciência, incluindo aí sua relação com a filosofia, tem certas particularidades que não devem ser ignoradas, caso se pretenda de fato compreender essa questão (ARAUJ O, 2009; ASH, 1980; BROOKS, 1997; GUILLIN, 2004; GUNDLACH, 2004a; 2004b; 2012; HATFIELD, 2012; NICOLAS, 2002; SCHMIDT, 1995; WILSON, 1990). Isso significa que a separação entre ambas ocorreu de modo distinto em diferentes países, o que nos obriga a abandonar o ideal de uma explicação geral e única para esse fato, e a buscar uma compreensão particular de acordo com cada contexto específico em questão.

Tendo em vista essa necessidade de uma contextualização específica para a historiografia da psicologia, o objetivo do presente artigo é fornecer os elementos centrais para a compreensão de um aspecto chave no desenvolvimento da psicologia alemã e de sua conflituosa relação com a filosofia: o Manifesto elaborado por filósofos alemães, em 1913, contra a ocupação de cátedras de filosofia por representantes da psicologia experimental. Simultaneamente, será fornecida uma tradução integral do texto original, seguida de algumas indicações sobre as suas consequências.

\section{O contexto geral: o desenvolvimento e a institucionalização da psicologia na Alemanha}

Para que possamos compreender corretamente o surgimento e a significação do Manifesto de 1913, é preciso situá-lo em seu contexto. Para tanto, porém, penso ser importante estabelecer aqui uma diferença entre o contexto geral e o contexto específico do mesmo. Por contexto geral, eu entendo o conjunto de ideias e eventos relacionados ao surgimento e à consolidação da psicologia como ciência e como disciplina na tradição alemã, incluindo aí a introdução da psicologia experimental nas universidades alemãs. Por 
contexto específico, eu entendo o fato singular que desencadeou uma reação coletiva de alguns filósofos, culminando na publicação do referido manifesto. Vejamos cada um deles separadamente.

É bem sabido que a concepção moderna de uma psicologia científica teve início na tradição filosófica alemã. No entanto, ao contrário do que diz a grande parte dos manuais de história da psicologia, isso não aconteceu na segunda metade do século XIX, a partir da fundação do laboratório de Wundt. Embora não seja fácil apontar com certeza uma data específica para o seu surgimento, há evidências mais do que suficientes de que já no século XVIII havia tanto uma concepção de psicologia como campo de conhecimento específico (p.ex., Christian Wolff) quanto uma série de pesquisas experimentais sobre processos psíquicos e disputas metodológicas acirradas (ARAUJ O, 2012; BRAUNS, 2002; HATFIELD, 1994; STURM, 2009, 2012; VIDAL, 2000), que continuaram a se desenvolver ao longo de todo o século XIX. Vale aqui notar que, neste momento, a universidade ainda não desempenhava um papel fundamental no desenvolvimento desta ciência, uma vez que muitos dos autores envolvidos tanto nas pesquisas quanto nas disputas estavam fora dela.

É nesse desenvolvimento da psicologia enquanto campo autônomo de investigação empírica que encontramos então as primeiras tensões com a filosofia. Mais especificamente, surge desde o início uma questão central, relacionada ao papel da psicologia no conhecimento filosófico. Wolff, por exemplo, colocava a psicologia empírica na base do seu sistema filosófico (WOLFF, 1728 [2006]). Por outro lado, Kant defendia a independência da filosofia em relação à psicologia, sobretudo no que diz respeito à lógica e à metafísica (KANT, 1781 [1998]). Ao longo do século XIX, essa questão reaparece constantemente nos debates, de forma que muitos filósofos vão tentar purificar a filosofia contra a sua "contaminação" com as ciências empíricas, reafirmando a irrelevância da psicologia para o conhecimento filosófico, como, p. ex., os neokantianos, Frege e Husserl. O ponto culminante dessa discussão é a assim chamada "querela do psicologismo" (Psychologismusstreit), que se tornou um verdadeiro campo de batalha entre psicólogos e filósofos alemães no final do século $X I X$ e início do século $X X$, com repercussões até os dias atuais (JACQUETTE, 2003; KAISER-EL-SAFTI; LOH, 2011; KUSCH, 1995; RATH, 1994; SCHMIDT, 1995).

Paralelamente ao desenvolvimento da psicologia como campo de investigação, acontece na Alemanha um fato inédito na cultura ocidental, a saber, a institucionalização da psicologia como disciplina obrigatória nas universidades alemãs. Isso se dá no âmbito de uma ampla reforma educacional implementada pelo governo da Prússia, no início do século XIX, que teve consequências profundas para a educação superior e o desenvolvimento da universidade alemã 
(NIPPERDAY, 1998; WEHLER, 2008). A partir de um decreto ministerial, em 1824, a psicologia passou a ser um conteúdo exigido obrigatoriamente no exame final dos professores ginasiais, formados pela Faculdade Filosófica ${ }^{1}$. Isso gerou, por sua vez, a necessidade de se oferecerem aulas de psicologia nas universidades e de contratação de professores para ministrar essas aulas. Curiosamente, porém, não foram criadas cadeiras específicas de psicologia, com seus respectivos detentores, para atender às novas demandas educacionais. Ao contrário, os próprios professores de filosofia tiveram que se encarregar de lecionar e supervisionar os conteúdos da nova disciplina. Isso significa que o surgimento da psicologia como disciplina não está atrelado a nenhuma investigação empírica ou experimental dos processos psíquicos, mas apenas a uma demanda institucional de formar professores para o ensino ginasial. Significa, além disso, que a institucionalização da psicologia enquanto disciplina vincula-a não só à Faculdade de Filosofia, mas principalmente às cadeiras e, consequentemente, aos docentes e professores de filosofia, uma vez que não foram criadas cátedras específicas para uma nova classe de especialistas (os psicólogos). Em terceiro lugar, a criação da psicologia enquanto disciplina não coincide inicialmente com a psicologia enquanto ciência, que era praticada por representantes de diversas disciplinas em outras faculdades ( $p$. ex., a Faculdade de Medicina) e também fora das universidades (GUNDLACH, 2004, 2012).

As reformas educacionais introduzidas na Alemanha do século XIX não afetaram apenas a estrutura dos currículos e de suas respectivas disciplinas. Outra consequência importante foi o surgimento e a proliferação dos institutos e laboratórios de pesquisa empírica, que deram às universidades alemãs um prestígio incomparável no século XIX, incluindo aí a Universidade de Leipzig (BLECHER; WIEMERS, 2004; RINGER, 2000). É neste momento que Wundt desempenha um papel fundamental. A criação, em 1879, do Instituto de Psicologia da Universidade de Leipzig, juntamente com o estabelecimento do Laboratório de Psicologia Experimental, foi também um fato inédito na cultura ocidental. Em primeiro lugar, não existia até então um Instituto de Psicologia com orçamento próprio para pesquisas empíricas nas Faculdades de Filosofia das universidades alemãs, uma vez que as pesquisas sobre tópicos psicológicos (sensação, percepção, etc.) eram feitas principalmente por fisiologistas nas Faculdades de Medicina. Por isso, o laboratório de Wundt, vinculado a uma cadeira de filosofia, foi uma verdadeira inovação. Além disso, foi uma iniciativa bem sucedida, na medida em que atraiu estudantes do mundo inteiro, tornando o Instituto de Psicologia da Universidade de Leipzig o primeiro centro internacional de formação de psicólogos (ARAUJ O, 2009). 
A criação de um instituto de psicologia vinculado à pesquisa experimental foi logo copiada por outras universidades alemãs. Depois de Leipzig, foram criados importantes institutos em Berlim, Göttingen e Würzburg, o que levou a uma rápida expansão e institucionalização da psicologia experimental na Alemanha (GEUTER, 1986). No entanto, é importante estarmos atentos para o fato de que essa institucionalização da psicologia experimental também não coincide com a institucionalização anterior da disciplina, já que nem todas as cadeiras de filosofia tinham institutos e laboratórios de psicologia a elas vinculados. Isso gerou um duplo desconforto. Em primeiro lugar, surgiu uma tensão entre os docentes da disciplina 'psicologia': de um lado, os representantes da nova psicologia experimental, que sabiam fazer pesquisa empírica e manipular instrumentos; de outro, os filósofos que ensinavam psicologia à maneira tradicional e não sabiam fazer nada disso. Para esse segundo grupo tornou-se cada vez mais difícil, devido às crescentes demandas metodológicas e técnicas, conseguir uma contratação para as cadeiras de filosofia associadas a esses novos institutos ou laboratórios de psicologia. Em segundo lugar, como esse avanço da psicologia experimental não vinha acompanhado da criação de cadeiras próprias para a área, a única possibilidade que os novos psicólogos experimentais tinham de conseguir uma colocação na universidade era ocupando mais uma cadeira de filosofia. A situação chegou a um ponto tal que, em 1913, os psicólogos experimentais já tinham conseguido mais de vinte por cento das cadeiras de filosofia das universidades alemãs (KUSCH, 1995). Até então, porém, a tensão ainda não tinha gerado uma revolta coletiva.

\section{O contexto específico: a ocupação da cátedra de filosofia na Universidade de Marburg}

A causa imediata para a publicação do Manifesto foi a assim chamada "querela da cátedra" (Lehrstuhlstreit). Em junho de 1912, o famoso filósofo neokantiano, Hermann Cohen, aposentou-se, deixando vaga a sua cadeira de filosofia na Universidade de Marburg. Segundo a sugestão do próprio Cohen e também de Paul Natorp, outro filósofo neokantiano, a vaga deveria ser ocupada pelo filósofo Ernst Cassirer. Ao invés disso, contudo, a Faculdade de Filosofia escolheu para o lugar de Cohen o psicólogo Erich Jaensch, que estudara com Ebbinghaus e fizera seu doutorado com George Müller em Göttingen (HOLZHEY, 1986, p. 21-22).

Em 12 de outubro de 1912, Natorp publicou um artigo em um dos jornais mais famosos da Alemanha - Frankfurter Zeitung - com o título de "A Herança Acadêmica de Hermann Cohen. Psicologia ou Filosofia?". Nesse artigo, ele criticou explicitamente a ocupação da 
cátedra de Cohen por um representante da psicologia experimental (NATORP, 1912).

No início de 1913, seis filósofos alemães - Heinrich Rickert (Freiburg), Paul Natorp (Marburg), Rudolf Eucken (Jena), Edmund Husserl (Göttingen), Alois Riehl (Berlin) e Wilhelm Windelband (Heidelberg) - iniciaram um movimento de protesto, elaborando uma declaração contra a ocupação de cadeiras de filosofia por representantes da psicologia experimental. Em seguida, enviaram-na a todas as Faculdades de Filosofia da Alemanha, Áustria e Suíça, angariando um total de 106 assinaturas de docentes e professores ${ }^{2}$. Em 26 de fevereiro de 1913, na reunião da Faculdade de Filosofia da Universidade de Leipzig, Wundt e outros importantes membros daquela Faculdade - como Karl Lamprecht, Georg Simmel e Eduard Spranger - tomaram conhecimento desse documento e resolveram, por sua vez, também expressar publicamente seus respectivos posicionamentos individuais, o que acabou gerando um extenso debate entre intelectuais alemães (KÖHNKE, 2005, p. 460-462 e 482485).

Em março de 1913, finalmente, o Manifesto foi publicado, primeiro na revista Logos, e posteriormente em diversos outros periódicos alemães ${ }^{3}$.

\section{O Manifesto e seus signatários}

O texto original do Manifesto, tal como publicado em Logos, traz o seguinte conteúdo:

Os abaixo-assinados, docentes de filosofia em instituições de ensino superior da Alemanha, Áustria e Suíça, vêem-se obrigados a fazer uma declaração, que se dirige contra a ocupação de cadeiras de filosofia por representantes da psicologia experimental.

O campo de trabalho da psicologia experimental tem se expandido de tal forma com o avanço altamente gratificante desta ciência, que ela há muito tempo é reconhecida como uma disciplina autônoma, cujo exercício demanda toda a energia de um pesquisador. Entretanto, não foram criadas para ela cadeiras independentes. Ao invés disso, cadeiras de filosofia têm sido repetidamente ocupadas por homens, cuja atividade é dedicada em grande parte ou exclusivamente à investigação experimental da vida mental. Na verdade, isso é compreensível quando se olha retrospectivamente para o início desta ciência, e era também provavelmente inevitável que ambas as disciplinas fossem representadas ao mesmo tempo por um único pesquisador. O desenvolvimento progressivo da psicologia experimental, contudo, gerou desconforto para todos os envolvidos. Sobretudo à filosofia, pela qual 0 interesse dos jovens estudantes está 


\begin{abstract}
constantemente crescendo, é causado um dano sensível através da retirada das cadeiras a ela dedicadas. Isso é ainda mais grave quando o campo da filosofia está cada vez mais se expandindo, e quando, exatamente em nossa época filosoficamente agitada, não se pode dar aos estudantes a oportunidade de se orientar cientificamente junto aos seus professores também sobre as questões gerais relacionadas à visão de mundo e à concepção de vida.

Dito tudo isso, os signatários consideram como sua obrigação apontar para as faculdades de filosofia e também para as autoridades educacionais as desvantagens decorrentes dessa situação para o estudo da filosofia e da psicologia. Em nome do interesse comum de ambas as ciências, deve-se atentar cuidadosamente para que a filosofia preserve seu lugar na vida acadêmica. Assim, a psicologia experimental deveria ser futuramente auxiliada apenas através da criação de cadeiras próprias; e em todos os lugares onde as velhas cadeiras de filosofia foram ocupadas por representantes da psicologia experimental, deve-se providenciar a criação de novas cadeiras filosóficas. (Rickert et al., 1913, p. 115-116)
\end{abstract}

Os 106 signatários do manifesto, que aparecem em ordem alfabética, de acordo com o sobrenome, são:

1) Prof. v. Aster (München); 2) Dr. Baensch (Straßburg i. E.); 3) Prof. Barth (Leipzig); 4) Prof. Bauch (Jena); 5) Dr. Bergmann (Leipzig); 6) Dr. Braun (Münster); 7) Prof. v. Brockdorff (Kiel); 8) Dr. Brunstädt (Erlangen); 9) Dr. Brunswig (München); 10) Dr. v. Bubnoff (Heidelberg); 11) Dr. Cassirer (Berlin); 12) Prof. Cohen (Marburg); 13) Prof. J. Cohn (Freiburg i. B.); 14) Prof. Cornelius (Frankfurt a. M.); 15) Prof. Deussen (Kiel); 16) Prof. Dinger (Jena); 17) Prof. Drews (Karlsruhe); 18) Dr. Eleutheropulos (Zürich); 19) Prof. Erhardt (Rostock); 20) Dr. Ehrenberg (Heidelberg); 21) Prof. Eucken (Jena); 22) Dr. Ewald (Wien); 23) Prof. Falckenberg (Erlangen); 24) Dr. A. Fischer (München); 25) Dr. Focke (Posen); 26) Prof. Freytag (Zürich); 27) Dr. Frischeisen-Köhler (Berlin); 28) Dr. Geiger (München); 29) Prof. Geyser (Münster); 30) Prof. Goedeckemeyer (Königsberg); 31) Prof. Goldstein (Darmstadt); 32) Dr. Gomperz (Wien); 33) Dr. Görland (Hamburg); 34) Dr. Groethuysen (Berlin); 35) Prof. Güttler (München); 36) Dr. Guttmann (Breslau); 37) Dr. Haberlin (Basel); 38) Dr. Hammacher (Bonn); 39) Dr. Hartmann (Marburg); 40) Prof. Heman (Basel); 41) Dr. Henning (Braunschweig); 42) Prof. Hensel (Erlangen); 43) Dr. Heyfelder (Tübingen); 44) Prof. Hönigswald (Breslau); 45) Prof. Husserl (Göttingen); 46) Dr. Jacoby (Greifswald); 47) Prof. Jerusalem (Wien); 48) Prof. Jodl (Wien); 49) Prof. Jöel (Basel); 50) Dr. Kabitz (Breslau); 51) Prof. Kinkel (Gießen); 52) Dr. Klemm (Leipzig); 53) Dr. Köster (München); 54) Dr. Kroner (Freiburg i. B.); 55) Dr. Kuntze (Berlin); 56) Prof. Lask (Heidelberg); 57) Prof. Lasson (Berlin); 58) Prof. Lehmann (Posen); 59) Prof. Leser (Erlangen); 60) Dr. Lessing 
(Hannover); 61) Dr. Linke (Jena); 62) Prof. G. F. Lipps (Zürich); 63) Prof. Medicus (Zürich); 64) Dr. Mehlis (Freiburg i. B.); 65) Dr. Menzel (Kiel); 66) Prof. Menzer (Halle); 67) Prof. Messer (Gießen); 68) Dr. Metzger (Leipzig); 69) Dr. Meyer (München); 70) Prof. Misch (Marburg); 71) Prof. Natorp (Marburg); 72) Dr. Nelson (Göttingen); 73) Dr. Nohl (Jena); 74) Prof. Pfänder (München); 75) Prof. v. d. Pfordten (Straßburg i. E.); 76) Prof. Rehmke (Greifswald); 77) Dr. Reinach (Göttingen); 78) Dr. Reininger (Wien); 79) Prof. Rickert (Freiburg i. B.); 80) Prof. Riehl (Berlin); 81) Prof. Ritter (Tübingen); 82) Dr. Ruge (Heidelberg); 83) Dr. Schlick (Rostock); 84) Prof. Schmekel (Greifswald); 85) Prof. F. A. Schmid (Heidelberg); 86) Prof. H. Schneider (Leipzig); 87) Dr. Schrempf (Stuttgart); 88) Prof. Schwarz (Greifswald); 89) Dr. Seidl (Zürich); 90) Dr. Siegel (Wien); 91) Prof. Simmel (Berlin); 92) Prof. Spitta (Tübingen); 93) Prof. Spitzer (Graz); 94) Prof. Spranger (Leipzig); 95) Prof. Tönnies (Kiel); 96) Prof. Uphues (Halle); 97) Dr. Utitz (Rostock); 98) Prof. Vaihinger (Halle); 99) Dr. Verweyen (Bonn); 100) Prof. Wahle (Czernowitz); 101) Prof. Wallaschek (Wien); 102) Dr. Weidenbach (Gießen); 103) Prof. Wentscher (Bonn); 104) Prof. Wernicke (Braunschweig); 105) Prof. Willmann (Prag); 106) Prof. Windelband (Heidelberg).

\section{As reações e consequências do Manifesto}

Como ressaltamos acima, ainda antes de sua publicação oficial, o documento foi primeiramente enviado às Faculdades de Filosofia na Alemanha, Áustria e Suíça, para que os docentes tomassem conhecimento. Portanto, antes também que saísse o número da revista Logos com o manifesto, alguns autores já haviam se manifestado publicamente em relação ao documento.

O primeiro deles foi Wundt, que tornou público, já em fevereiro de 1913, seu escrito Die Psychologie im Kampf ums Dasein (A Psicologia em Luta pela Existência). De acordo com Wundt, a separação entre as disciplinas seria ruim para ambos os lados. Por isso, criticou tanto os filósofos quanto os psicólogos que queriam a separação. Ou seja, ao contrário do que muitas vezes se afirma na historiografia da psicologia, Wundt não foi responsável pela independência da psicologia em relação à filosofia, simplesmente por que ele defendia que a formação do psicólogo estava imersa em questões filosóficas, das quais ele jamais poderia escapar (WUNDT, 1913 [1921]). Isso pode ser mais bem ilustrado na seguinte passagem, que ele utiliza para criticar a proposta de separação:

É curioso notar que, nestas disputas sobre o direito da psicologia alcançar o status de uma ciência autônoma, [...] uma questão permanece intocada, embora devesse ser 
considerada a mais crucial de todas, a saber: em que medida o psicólogo em geral pode prescindir da filosofia e abrir mão, sempre que ele quiser se aprofundar nos seus próprios problemas, do auxílio das reflexões filosóficas que [...] ele mesmo deve extrair do seu próprio trabalho? (WUNDT, 1913 [1921], p. 517).

Mas Wundt não foi o único a se manifestar. Karl Marbe, um de seus ex-alunos, publicou uma severa crítica ao manifesto. Segundo ele, o ataque à psicologia experimental era uma estratégia camuflada, pois o que eles queriam é acabar com a psicologia como um todo: "A campanha é dirigida de fato contra a moderna psicologia", afirmou ele (MARBE, 1913, p. 16). Além disso, Marbe criticou duramente os filósofos signatários, afirmando sua falta de competência para julgar questões relacionadas à psicologia (MARBE, 1913, p. 22).

O Manifesto de 1913 desencadeou, na verdade, uma onda de críticas, réplicas e tréplicas. Não só psicólogos e filósofos, mas também representantes das Geisteswissenschaften em geral tomaram posição em favor ou contra o manifesto, como o historiador Karl Lamprecht e o sociólogo Georg Simmel (LAMPRECHT, 1913a, 1913b; SIMMEL, 1913). Infelizmente não é possível apresentar aqui todos os seus desdobramentos ${ }^{4}$.

Com o início da Primeira Guerra Mundial, em 1914, o debate perdeu sua força inicial e desapareceu da dimensão pública. No entanto, ele jamais desapareceu do imaginário dos filósofos e psicólogos alemães, deixando uma marca indelével na posterior cultura acadêmica alemã. Apenas como ilustração, vale aqui lembrar o posicionamento da Faculdade de Filosofia da Universidade de Jena, quase dez anos depois, contra o projeto ministerial de criação de uma cadeira de psicologia experimental (ECKARDT, 1996).

Foi dessa relação tensa - iniciada já no século XVIII e presente ao longo de todo o século XIX, mas agravada pelo manifesto de 1913 que surgiu a separação institucional entre a filosofia e a psicologia nas universidades alemãs, a partir do final da primeira metade do século XX. A independência institucional, por sua vez, levou a um distanciamento intelectual mútuo ainda maior. Não deve causar surpresa, pois, o fato de até hoje, nas universidades alemãs, não haver praticamente nenhum contato entre filósofos e psicólogos, ainda que ambos os lados não tenham mais consciência das origens dessa separação.

\section{Considerações finais}

A inserção do Manifesto de 1913 entre as fontes primárias para investigação do desenvolvimento histórico da psicologia nos permite tirar ao menos duas lições para a historiografia futura da psicologia. 
Em primeiro lugar, o Manifesto de 1913 nos obriga a considerar que a separação institucional entre a filosofia e a psicologia não ocorreu de forma homogênea na história, mas que, ao contrário, a relação entre ambas as disciplinas assume contornos específicos do seu contexto cultural. Isso significa que a tensão extrema que caracteriza o caso alemão não deve ser adotada como norma geral para outros países, como os EUA, a França e o Brasil. É bem possível que nesses outros contextos essa relação tenha se desenvolvido de forma diferente, ainda que por períodos bem definidos.

Em segundo lugar, o estudo tanto do Manifesto de 1913 quanto das relações entre filósofos e psicólogos na tradição alemã nos ensina uma lição ainda mais geral: a de que o desenvolvimento e a consolidação da psicologia científica não podem ser vistos a partir de uma perspectiva historiográfica puramente internalista ou externalista, uma vez que há aí nitidamente uma mistura de fatores tanto internos (lógicos, metodológicos, filosóficos) quanto externos (sociais, políticos, institucionais). O episódio aqui em questão serve como excelente exemplo da interação entre ambos os tipos de fatores. Em outras palavras, ele exige a distinção, mas ao mesmo tempo um equilíbrio entre as perspectivas internalista e externalista. Desse equilíbrio depende, a nosso ver, a qualidade da historiografia futura da psicologia científica, pelo menos no que diz respeito às relações históricas entre filosofia e psicologia.

\section{Referências}

ARAUJO, S. F. Wilhelm Wundt e a fundação do primeiro Centro Internacional de Formação de Psicólogos. Temas em Psicologia, Ribeirão Preto, v. 17, n. 1, p. 9-14, 2009.

. O lugar de Christian Wolff na história da psicologia. In: S. F.

ARAUjO (Org.), História e Filosofia da Psicologia: Perspectivas Contemporâneas. Juiz de Fora: Ed. UFJF, 2012, p. 67-86.

ASH, M. G. Experimental psychology in Germany before 1914: aspects of an academic identity problem. Psychological Research, Carbondale, v. 42, p. 75-86, 1980.

BLECHER, J.; WIEMERS, G. Die Universität Leipzig 1409-1943. Erfurt: Stutton Verlag, 2004.

BRAUNS, H.-P. Methodenfrage der Psychologie im 18. Jahrhundert. Psychologie und Geschichte, Hagen, v. 10, n. 3-4, p. 178-199, 2002.

BROOKS, J. I. The eclectic legacy. Academic philosophy and the human sciences in nineteenth-century France. Newark: University of Delaware Press, 1997.

ECKARDT, G. D. Psychologie im Spannungsfeld wissenschaftspolitischer und hochschulpolitischer 
Auseinandersetzungen: der Thüringer Hochschulkonflikt 1923. In: GUNDLACH, H. (Hg.), Untersuchungen zur Geschichte der Psychologie und der Psychotechnik. München; Wien: Profil (Passauer Schriften zur Psychologiegeschichte, 11), 1996, p. 289300.

EISENMEYER, J. Die Psychologie und ihre zentralle Stellung in der Philosophie. Halle: Niemeyer, 1914.

FISCHER, A. Philosophie und Psychologie: eine prinzipielle Betrachtung zu einer aktuellen der Fortbildung der Hochschule. Die deutsche Schule, Leipizg, v. 17, p. 338-347, 1913.

FRISCHEISEN-KÖHLER, M. Philosophie und Psychologie. Die Geisteswissenschaften, Leipzig, v. 1, n. 14, p. 371-373, 1913 a.

FRISCHEISEN-KÖHLER, M. Philosophie und Psychologie. Die Geisteswissenschaften, Leipzig, v. 1, n. 15, 400-403, 1913b. GEIGER, M. Philosophie und Psychologie an den deutschen Universitäten. Süddeutsche Monatshefte, München, v. 10, n. 2, p. 752-755, 1913.

GEUTER, U. Daten zur Geschichte der deutschen Psychologie. Band 1. Göttingen: Hogrefe, 1986.

GUILLIN, V. Théodule Ribot's ambiguous positivism: philosophical and epistemological strategies in the founding of French scientific psychology. Journal of the History of the Behavioral Sciences, Malden, v. 40, n. 2, p. 165-181, 2004.

GUNDLACH, H. Reine Psychologie, Angewandte Psychologie und die Institutionalisierung der Psychologie. Zeitschrift für Psychologie, Göttingen, v. 212, n. 4, p. 183-199, 2004.

A psicologia como ciência e como disciplina: o caso da Alemanha. In: ARAUJO, S. F. (Org.). História e filosofia da psicologia: perspectivas contemporâneas. Juiz de Fora: Ed. UFJF, 2012, p. 133-165.

HATFIELD, G. Psychology as a natural science in the eighteenth century. Revue de Synthèse, Paris, v. 115, p. 375-391, 1994.

Psicologia, filosofia e ciência cognitiva: reflexões sobre a história e a filosofia da psicologia experimental. In: ARAUJO, S. F. (Org.). História e filosofia da psicologia: perspectivas contemporâneas. Juiz de Fora: Ed. UFJ F, 2012, p. 223-259.

HILLEBRAND, F. Die Aussperrung der Psychologen. Ein Wort der Klärung. Leipzig: J ohann Ambrosius Barth, 1913.

HOLZHEY, H. Cohen und Natorp. 2 Bände. Basel/Stuttgart: Schwabe \& Co., 1986.

J ACQUETTE, D. (Ed.). Philosophy, psychology and psychologism: critical and historical readings on the psychological turn in philosophy. Dordrecht: Springer, 2003.

KAISER-EL-SAFTI, M.; LOH, W. Die Psychologismus-Kontroverse. Göttingen: Vandenhoeck \& Ruprecht, 2011. 
KANT, I. (1781). Kritik der reinen Vernunft. Ed. by J. Timmermann. Hamburg: Meiner, 1998.

KÖHNKE, K.C. Editorischer Bericht. In: (Hg.) Georg Simmel Gesamtausgabe. Band 17 Frankfurt a.M.: Sührkamp. 2005, p. 446513.

$\mathrm{KUSCH}, \mathrm{M}$. Psychologism: A case study in the sociology of philosophical knowledge. London: Routledge, 1995.

LAMPRECHT, K.Eine Gefahr für die Geisteswissenschaften. Die Zukunft, Berlin, v. 83, p. 16-24, 1913a.

. Eine Gefahr für die Geisteswissenschaften. Antwort an Herrn Prof. Dr. Georg Simmel. Die Zukunft, Berlin, v. 83, p. 421-429, 1913b.

MARBE, K. Die Aktion gegen die Psychologie. Eine Abwehr. Leipzig: B. G. Teubner, 1913.

NATORP, P. Das akademische Erbe Hermann Cohens. Psychologie oder Philosophie? Frankfurter Zeitung, Frankfurt, v. 57, n. 283, p. 1-2, 12 out. 1912.

$\overline{1} \overline{7} \overline{6}-\overline{2} 02,1913$.

Philosophie und Psychologie. Logos, Tübingen, v. 4, p.

NICOLAS, S. Histoire de la psychologie française: naissance d'une nouvelle science. Paris: In Press Editions, 2002.

NIPPERDEY, T. Deutsche Geschichte 1800-1866. Bürgerwelt und starker Staat. München: Beck, 1998.

PAULSEN, F. The German universities: their character and historical development. New York: Macmillan and Co, 1895.

RATH, M. Der Psychologismusstreit in der deutschen Psychologie. Freiburg; München: Alber, 1994.

RICKERT, R. Zur Besetzung der philosophischen Professuren mit Vertretern der experimentellen Psychologie. Frankfurter Zeitung, Frankfurt, p. 1-2, 4 mar. 1913.

RICKERT, H. et al. Erklärung. Logos, Tübingen, v. 4, p. 115-116, 1913.

RINGER, F. O declínio dos mandarins alemães. São Paulo: EDUSP,2000.

SCHMIDT, N. Philosophie und Psychologie. Trennungsgeschichte, Dogmen und Perspektiven. Reinbeck: Rowohlt, 1995.

SIMMEL, G. An Herrn Prof. Karl Lamprecht. Die Zukunft, Berlin, v. 83, p. 230-234, 1913.

SPRANGER, E. Zum Streit um die Psychologie. Deutsche Literaturzeitung, Berlin, v. 34, n. 12, p. 708-715, 1913.

STURM, T. Kant und die Wissenschaften vom Menschen. Paderborn: Mentis, 2009.

Há algum problema com a psicologia matemática no século dezoito? Um novo olhar sobre o velho argumento de Kant. In: ARAUJO, S. F. (Org.). História e filosofia da psicologia: 
perspectivas contemporâneas. Juiz de Fora: Ed. UFJF, 2012, p. 87-132.

VIDAL, F. The eighteenth century as 'century of psychology'. Jahrbuch für Recht und Ethik/ Annual Review of Law and Ethics, Berlin, v. 8, p. 407-434, 2000.

WEHLER, H.-U. Deutsche Gesellschaftsgeschichte 1700-1815. 4. Aufl. München: Beck, 2008.

WILSON, D. J. Science, community, and the transformation of american philosophy, 1860-1930. Cicago: University of Chicago Press, 1990.

WOLFF, C. (1728). Einleitende Abhandlung über Philosophie im allgemeinen. Stuttgart/Bad Cannstatt: Frommann-Holzboog, 2006. WUNDT, W. (1913). Die Psychologie im Kampf ums Dasein. In: Kleine Schriften. Band. I I I. Leipzig: Engelmann, 1921, p. 515-543.

\section{Endereço para correspondência \\ Saulo de Freitas Araujo}

Universidade Federal de Juiz de Fora

Instituto de Ciências Humanas/Departamento de Psicologia

Rua J osé Lourenço Kelmer, s/n - Campus Universitário

Bairro São Pedro - CEP: 36036-900 - Juiz de Fora - MG

Tel: (32) 3272-1595 / 9944-1573

Endereço eletrônico: saulo.araujo@ufjf.edu.br

Recebido em: 25/01/2012

Aceito para publicação em: 27/08/2012

Acompanhamento do processo editorial: Ana Maria Jacó Vilela

\section{Notas}

* Professor Doutor do Departamento de Psicologia e do Programa de Pós-Graduação em Psicologia da Universidade Federal de Juiz de Fora, J uiz de Fora, Brasil.

1 A universidade alemã era formada por três faculdades superiores (Teologia, Direito e Medicina), que ofereciam uma formação para uma profissão específica (teólogo, advogado, médico), e uma faculdade inferior (Filosofia), que servia apenas de propedêutica às faculdades superiores (PAULSEN, 1895). A partir de 1810, como consequência das reformas educacionais, ela ganhou uma nova função: a formação de um novo tipo de profissional, a saber, o professor ginasial. No fim de sua formação, o candidato deveria prestar um exame estatal, que incluía diversas matérias (filosofia, pedagogia, etc.). A partir do decreto de 1824, a psicologia passou a fazer parte das matérias obrigatórias cobradas neste exame (GUNDLACH, 2004).

2 Nas universidades alemãs, a carreira do magistério superior é bem diferente da carreira no Brasil. Professor é um título - o mais alto da carreira - outorgado apenas ao docente que, indicado pelos seus pares e referendado pelo Ministro da Educação, passa a ser o detentor de uma determinada cátedra em uma Faculdade (p. ex., a cátedra de História da Filosofia). Com isso, ele passa a gozar de estabilidade, salário diferenciado, alguns privilégios institucionais - como verbas específicas para a compra de livros e a autorização para contratar assistentes de pesquisa - e um altíssimo prestígio social. Mas isso só acontece com uma pequena parte dos docentes, uma vez que há poucas cátedras para muitos pretendentes. Uma boa parte do quadro docente é composta por Privatdozenten, que também podem oferecer cursos regulares e fazer pesquisa, mas não têm estabilidade e nem 
recebem um salário fixo do Estado, além de não poderem votar e nem participar das reuniões oficiais da Faculdade. Existe, pois, uma hierarquia institucional bem acentuada, de forma que nem todo docente é um professor (PAULSEN, 1895; RINGER, 2000).

${ }^{3}$ Os outros periódicos que também publicaram o manifesto em 1913 foram: Archiv für Geschichte der Psychologie, v. 26, n. 3, p. 399-400; Archiv für systematische Philosophie, v. 19, n. 2, p. 273-274; Kant-Studien, v. 18, n. 3, p. 306-307; Vierteljahrsschrift für wissenschaftliche Philosophie und Soziologie, v.37, n. 2, p. 341-343.

4 Vale, no entanto, destacar os principais escritos relacionados ao manifesto: Eisenmeyer, 1914; Fischer, 1913; Frischeisen-Köhler, 1913; Geiger, 1913; Hillebrand, 1913; Lamprecht, 1913a, 1913b; Marbe, 1913; Natorp, 1913; Rickert, 1913; Simmel, 1913; Spranger, 1913; Wundt, 1913. 